\title{
Attractivity and stability in the competitive systems of PDEs of Kolmogorov type
}

\author{
Joanna Balbus \\ Institute of Mathematics and Computer Science, Wrockaw University of Technology, \\ Wybrzeże Wyspiańskiego 27, PL-50-370 Wroctaw, Poland
}

\begin{abstract}
In this paper we consider $N$-species nonautonomous competitive systems of partial differential equations of Kolmogorov type. Under the Neumann boundary conditions we give a sufficient condition for the system to be uniformly stable and globally attractive.
\end{abstract}

Keywords: Reaction - diffusion system, upper average, lower average, persistence, permanence, Lyapunov functional, global attractivity, stability.

\section{Introduction}

In this paper we consider Kolmogorov systems of reaction-diffusion parabolic partial differential equations (PDEs)

$$
\frac{\partial u_{i}}{\partial t}=\Delta u_{i}+f_{i}\left(t, x, u_{1}, \ldots, u_{N}\right) u_{i} \quad t>0, x \in \Omega, 1 \leq i \leq N .
$$

From the biological viewpoint this problem represents a model of population growth where $u_{i}(t, x)$ is the density of the $i$-th species at time $t$ and spatial location $x \in \bar{\Omega}, \Omega \subset R^{n}$ is a bounded habitat, $f_{i}\left(t, x, u_{1}, \ldots, u_{N}\right)$ is the local per capita growth rate of the $i$-th species. The system is usually considered with appropriate boundary conditions, either Dirichlet or Neumann, or Robin boundary conditions. In this paper we investigate the system under the Neumann boundary conditions. We deal with competitive systems. It means that the derivatives $\frac{\partial f_{i}}{\partial u_{j}}, 1 \leq i, j \leq N$ are nonpositive.

One of the major problems in models of population growth are permanence and stability. The authors of many papers focus on finding necessary or sufficient conditions for permanence or stability in models which are described by ordinary or partial differential equations. Permanence means that any positive solution of the model has all its components, for large $t$, bounded away from zero and the lower bound is independent of the solution. Global attractivity states that the

Email address: joanna.balbus@pwr.wroc.pl (Joanna Balbus)

Preprint submitted to Elsevier 
difference of any two positive solutions tends to zero as $t$ goes to infinity. In [1] S. Ahmad and A.C. Lazer, using the upper and lower averages of a function, found sufficient conditions for permanence and global attractivity of the competitive $N$ species Lotka-Volterra system of ODEs. In [6] we extended their result for the $N$ species competitive Kolmogorov system of ODEs. In terms of the upper and lower averages of a function we found sufficient conditions for such a system to be permanent and globally attractive.

In [5] the authors considered two species Lotka - Volterra system of PDEs. They investigated behavior of this system not only as $t$ goes to infinity, but in the "pullback" sence. It means that we are starting with a fixed initial condition further and further back in time. They introduced a notion "pullback permanence" which has an interesting biological interpretation. When two species have already been competing for a long time then neither species will have died out. Their densities are bounded below in a uniform way, no matter how long this population has been running.

In [2] we investigate the $N$ species competitive Kolmogorov system of PDEs. Using the methods of supersolutions and subsolutions for parabolic PDEs we found sufficient conditions for permanence in such systems.

To establish the global attractivity many authors use appropriate Lyapunov function in the case of ODE or Lyapunov functional in the case of PDE (see for example [1], [6], [7], [8], [9]). In [3, 4] K. Gopalsamy dealt with, respectively, periodic and almost periodic solution of a Lotka - Volterra system of ODEs. A. Tineo [9] considered the nonautonomous Kolmogorov system of ODEs

$$
u_{i}^{\prime}=f_{i}(t, u) u_{i} \quad 1 \leq i \leq N,
$$

where $f: \mathbb{R} \times \mathbb{R}_{+}^{N} \rightarrow \mathbb{R}^{N}$ is a continuous function and $\mathbb{R}_{+}^{N}$ is the nonnegative orthant $\mathbb{R}_{+}^{N}=\left\{u \in \mathbb{R}^{N}: u_{i} \geq 0,1 \leq i \leq N\right\}$. He found a sufficient condition for the system ( $\mathrm{K}$ ) to be globally attractive. In [6] we considered system (K) with slightly weaker assumptions for the function $f$ than those $f$ in [9]. In [8] Sze-Bi Hsu examined Lyapunov functions (or functionals) for various ecological models which take form of ODE systems (or reaction-diffusion PDE systems). First the author constructed Lyapunov functions for the predator prey system and then constructed Lyapunov functionals for the corresponding reaction-diffusion PDE system. Sze-Bi Hsu considered the models in which the reaction term is independent on $t$ and $x$.

In [10] A. Tineo and J. Riviero dealt with the two species nonautonomous competitive Lotka-Volterra system with diffusion. They resigned from the Lyapunov function (functional) to establish of attractivity of this system. They used a powerful tool which is the iteration monotone methods.

Usually the same conditions which guarantee permanence in the ODE model of population growth ensure that the model is globally attractive ( see for example [1], [6]). But when we try to prove that a system of Kolmogorov type of PDEs is globally attractive then some difficulties occur. Firstly, we can prove that the system is globally attractive only under the Neumann boundary conditions. Secondly, besides the conditions which guarantee the permanence we 
need additional inequalities which contain the parameters which occur in the definition of permanence. Even in the case of two species problem it is a difficult to obtain sufficient conditions which guarantee both permanence and global attractivity of Kolmogorov system of PDEs. One can try to introduce a partial ordering. The idea is that we can compare solutions of our system of PDE with the functions which are independent on $x$ in a way that when we apply partial ordering our solutions of PDE are between the functions independent on $x$. A difficulty occurs when we let the parameter which is a spatial location to be arbitrary.

In this paper we find sufficient conditions for $N$ - species system of PDEs of Kolmogorov type under the Neumann boundary conditions to be globally attractive. Conditions which we obtained are stronger than those guaranteeing perrmanence. Assumptions for a function $f$ in this paper is the same as in [2].

This paper is organized as follows.

In Section 2 we present basic assumptions and definitions.

In Section 3 we formulate the main theorem of this paper. By Lyapunov functional some sufficient conditions are obtained to guarantee attractivity and uniform stability Kolmogorov system of PDEs.

In Section 4 we give some remarks.

\section{Preliminaries}

We consider a nonautonomous system of PDEs of Kolmogorov type

$$
\begin{cases}\frac{\partial u_{i}}{\partial t}=\Delta u_{i}+f_{i}\left(t, x, u_{1}, \ldots, u_{N}\right) u_{i}, & t>0, x \in \Omega, i=1, \ldots, N \\ \mathcal{B} u_{i}=0, & t>0, x \in \partial \Omega, i=1, \ldots, N,\end{cases}
$$

where $\Omega \subset \mathbb{R}^{n}$ is a bounded domain with a sufficiently smooth boundary $\partial \Omega, \Delta$ is the Laplace operator on $\Omega$,

$$
\Delta=\frac{\partial^{2}}{\partial x_{1}^{2}}+\cdots+\frac{\partial^{2}}{\partial x_{n}^{2}},
$$

and $\mathcal{B}$ is the boundary operator of the Neumann type

$$
\mathcal{B} u_{i}=\frac{\partial u_{i}}{\partial \nu} \quad \text { on } \partial \Omega,
$$

where $\nu$ denotes the unit normal vector on $\partial \Omega$ pointing out of $\Omega$.

Now we make the following assumptions

(A1) (see [2]) $f_{i}:[0, \infty) \times \bar{\Omega} \times[0, \infty)^{N} \rightarrow \mathbb{R}(1 \leq i \leq N)$, as well as their first derivatives $\partial f_{i} / \partial t(1 \leq i \leq N), \partial f_{i} / \partial u_{j}(1 \leq i, j \leq N)$, and $\partial f_{i} / \partial x_{k}$ $(1 \leq i \leq N, 1 \leq k \leq n)$, are continuous.

(A1) guarantees that for any regular initial function $u_{0}(x)=\left(u_{01}, \ldots, u_{0 N}\right)$, $x \in \Omega$ where $u_{0 i} \geq 0(1 \leq i \leq N, x \in \Omega)$ there exists a unique maximally 
defined solution $u(t, x)=\left(u_{1}(t, x), \ldots, u_{N}(t, x)\right)$ of $(\underline{\mathbb{R}}),(t, x) \in\left[0, \tau_{\max }\right) \times \bar{\Omega}$, where $\tau_{\max }>0$, satisfying the initial condition $u(0, x)=u_{0}(x)$. Moreover, the solution of system (R) is classical.

$(\mathrm{A} 2)\left(\right.$ see [2]) Functions $\left[[0, \infty) \times \bar{\Omega} \ni(t, x) \mapsto f_{i}(t, x, 0, \ldots, 0) \in \mathbb{R}\right], 1 \leq i \leq$ $N$, are bounded.

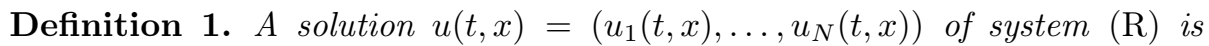
positive if $u_{i}(t, x)>0$ for all $i=1, \ldots, N, x \in \Omega, t>0$.

Definition 2. System $(\underline{\mathbb{R}})$ is permanent if there exist positive constants $\underline{\delta}, \bar{\delta}$ such that for any positive solution $u(t, x)=\left(u_{1}(t, x), \ldots, u_{N}(t, x)\right)$ there exists $T=T(u) \geq 0$ with the property

$$
\underline{\delta} \leq u_{i}(t, x) \leq \bar{\delta}
$$

for all $t \geq T, x \in \bar{\Omega}, i=1, \ldots, N$.

Definition 3. System ( $\mathrm{R}$ ) is globally attractive if any two positive solutions $u(t, x)=\left(u_{1}(t, x), \ldots, u_{N}(t, x)\right)$ and $v(t, x)=\left(v_{1}(t, x), \ldots, v_{N}(t, x)\right)$ of system (R) satisfy

$$
\lim _{t \rightarrow \infty}\left(u_{i}(t, x)-v_{i}(t, x)\right)=0
$$

for $1 \leq i \leq N$, uniformly in $x \in \bar{\Omega}$.

Definition 4. System $(\mathbb{R})$ is uniformly stable on $\left[t_{1}, \infty\right), t_{1}>0$ if for every $\varepsilon>0$ there is a $\delta>0$ so that for any two positive solutions $u(t, x)=$ $\left(u_{1}(t, x), \ldots, u_{N}(t, x)\right)$ and $v(t, x)=\left(v_{1}(t, x), \ldots, v_{N}(t, x)\right)$ if $t_{2} \in\left[t_{1}, \infty\right)$ and $\left\|u\left(t_{2}, x\right)-v\left(t_{2}, x\right)\right\|<\delta$ then $\|u(t, x)-v(t, x)\|<\varepsilon$ for $t \geq t_{2}$.

The next assumption is

(A3)(see [2]) There exist $\underline{b}_{i i}>0$ such that $\frac{\partial f_{i}}{\partial u_{i}}(t, x, u) \leq-\underline{b}_{i i}$ for all $t \geq 0$, $x \in \bar{\Omega}, u \in[0, \infty)^{N}, 1 \leq i \leq N$.

Define

$$
B(\varepsilon):=\left[0, \frac{\bar{a}_{1}}{\underline{b}_{11}}+\varepsilon\right] \times \cdots \times\left[0, \frac{\bar{a}_{N}}{\underline{b}_{N N}}+\varepsilon\right], \quad \varepsilon \geq 0 .
$$

(A4)(see [2]) $\partial f_{i} / \partial u_{j}, 1 \leq i, j \leq N$, are bounded and uniformly continuous on each set $[0, \infty) \times \bar{\Omega} \times B$.

For $1 \leq i, j \leq N$ define

$$
\bar{b}_{i j}(\epsilon):=\sup \left\{-\frac{\partial f_{i}}{\partial u_{j}}(t, x, u): t \geq 0, x \in \bar{\Omega}, u \in B(\varepsilon)\right\} .
$$

and $\bar{b}_{i j}(0):=\bar{b}_{i j}$. 
Assumptions (A3) and (A4) imply that $\bar{b}_{i j}(\epsilon) \geq 0,1 \leq i, j, \leq N$, and $\bar{b}_{i i}(\epsilon)>$ $0,1 \leq i \leq N$, whereas it follows from (A5) that $\bar{b}_{i j}(\epsilon)<\infty$, and $\lim _{\epsilon \rightarrow 0^{+}} \bar{b}_{i j}(\epsilon)=$ $\bar{b}_{i j}$, for $1 \leq i, j \leq N$.

Recall that in [2] we found average conditions for system $(\mathbb{R})$ to be permanent. The average conditions for system $(\underline{\mathbb{R}})$ under the Neumann boundary conditions are

$$
m\left[f_{i}\right]>\sum_{\substack{j=1 \\ j \neq i}}^{N} \frac{\bar{b}_{i j} M\left[f_{j}\right]}{\underline{b}_{j j}}
$$

where

$$
m\left[f_{i}\right]:=\liminf _{t-s \rightarrow \infty} \frac{1}{t-s} \int_{s}^{t} \min _{x \in \bar{\Omega}} f_{i}(\tau, x, 0, \ldots, 0) d \tau
$$

and

$$
M\left[f_{i}\right]:=\limsup _{t-s \rightarrow \infty} \frac{1}{t-s} \int_{s}^{t} \max _{x \in \Omega} f_{i}(\tau, x, 0, \ldots, 0) d \tau .
$$

\section{Main Theorem}

In this section we state the main theorem of this paper and with the help of a Lyapunov functional we prove attractivity and stability of system $(\underline{R})$.

Theorem 3.1. Assume (A1) - (A5) and (AC). Let $\underline{\delta}, \bar{\delta} \geq 0$ be such that for each $1 \leq i \leq N$

$$
\underline{\delta}_{i i}>\sum_{\substack{j=1 \\ j \neq i}}^{N} \bar{\delta} \bar{b}_{j i}
$$

Suppose that $u(t, x)=\left(u_{1}(t, x), \ldots, u_{N}(t, x)\right)$ and $v(t, x)=\left(v_{1}(t, x), \ldots, v_{N}(t, x)\right)$ be two positive solutions of system $(\underline{\mathrm{R}})$ such that $\underline{\delta} \leq u_{i}(t, x), v_{i}(t, x) \leq \bar{\delta}$ for sufficiently large $t$ and all $x \in \bar{\Omega}$. Then there exist positive constants $Z, \gamma$ and $\tilde{T} \geq 0$ such that for $i=1, \ldots, N$ and $t \geq \tilde{T}$ one has

$$
\sum_{i=1}^{N} \sup _{x \in \bar{\Omega}}\left|u_{i}(t, x)-v_{i}(t, x)\right| \leq Z \sum_{i=1}^{N} \sup _{x \in \bar{\Omega}}\left|u_{i}(\tilde{T}, x)-v_{i}(\tilde{T}, x)\right| \cdot \exp (-\gamma(t-\tilde{T})),
$$

In particular system $(\underline{\mathbb{R}})$ is globally attractive and uniformly stable.

Proof. Fix two positive solutions $u(t, x)=\left(u_{1}(t, x), \ldots, u_{N}(t, x)\right)$ and $v(t, x)=$ $\left(v_{1}(t, x), \ldots, v_{N}(t, x)\right)$ of system $(\underline{\mathbb{R}})$. By assumption (A5) we can take $\varepsilon>0$ such that

$$
\underline{\delta}_{i} \underline{b}_{i i}>\sum_{\substack{j=1 \\ j \neq i}}^{N} \bar{\delta}_{j} \bar{b}_{i j}(\varepsilon) \text { for all } i=1, \ldots, N .
$$


Let $t_{0}>0$ be such that $u(t, x), v(t, x) \in B(\varepsilon)$ and $\underline{\delta} \leq u_{i}(t, x), v_{i}(t, x) \leq \bar{\delta}$ for all $t \geq t_{0}, x \in \bar{\Omega}, 1 \leq i \leq N$. By a result in matrix theory (see [7, Section 5]) we know that there exist $\alpha_{1}, \ldots, \alpha_{N}>0$ such that

$$
\alpha_{i} \underline{\delta}_{i i}>\sum_{\substack{j=1 \\ j \neq i}}^{N} \alpha_{j} \bar{\delta}_{j i}(\varepsilon), \quad 1 \leq i \leq N
$$

Denote

$$
\Theta(t)=\sum_{i=1}^{N} \alpha_{i} \Theta_{i}(t), \text { where } \Theta_{i}(t):=\sup _{x \in \bar{\Omega}}\left|\ln \frac{u_{i}(t, x)}{v_{i}(t, x)}\right| .
$$

Fix $1 \leq i \leq N$. We prove that there exists $\tilde{T} \geq t_{0}$ such that

$$
D^{+} \Theta_{i}(t) \leq-\underline{\delta}_{i i} \Theta_{i}(t)+\bar{\delta} \sum_{\substack{j=1 \\ j \neq i}}^{N} \bar{b}_{i j}(\varepsilon) \Theta_{j}(t), \quad t \geq \tilde{T} .
$$

where $D^{+} \Theta$ denotes the upper derivative of $\Theta$.

We take for $\tilde{T}$ any number $\geq t_{0}$. For $t \geq \tilde{T}$ denote $\bar{\Omega}^{(i)}(t)$ to be the set of the points $x^{(i)} \in \bar{\Omega}$ such that the supremum of the function $\left|\ln \frac{u_{i}(t, x)}{v_{i}(t, x)}\right|$ is realized. Then $\bar{\Omega}^{(i)}(t)=\bar{\Omega}_{+}^{(i)}(t) \cup \bar{\Omega}_{-}^{(i)}(t), \bar{\Omega}_{+}^{(i)}(t) \cap \bar{\Omega}_{-}^{(i)}(t)=\emptyset$, where

$$
\bar{\Omega}_{+}^{(i)}(t)=\left\{x^{(i)} \in \bar{\Omega}^{(i)}(t): u_{i}\left(t, x^{(i)}\right)>v_{i}\left(t, x^{(i)}\right)\right\}
$$

and

$$
\bar{\Omega}_{-}^{(i)}(t)=\left\{x^{(i)} \in \bar{\Omega}^{(i)}(t): u_{i}\left(t, x^{(i)}\right)<v_{i}\left(t, x^{(i)}\right)\right\}
$$

Therefore by (3.2)

$$
\begin{aligned}
D^{+} \Theta_{i}(t) \leq \max \{ & \sup \left\{\frac{\partial}{\partial t} \ln \frac{u_{i}\left(t, x^{(i)}\right)}{v_{i}\left(t, x^{(i)}\right)}: x^{(i)} \in \bar{\Omega}_{+}^{(i)}(t)\right\}, \\
& \left.\sup \left\{\frac{\partial}{\partial t} \ln \frac{v_{i}\left(t, x^{(i)}\right)}{u_{i}\left(t, x^{(i)}\right)}: x^{(i)} \in \bar{\Omega}_{-}^{(i)}(t)\right\}\right\} .
\end{aligned}
$$

Denote $\kappa_{i}:=\Theta_{i}(t)$. Now we consider four (not mutually exclusive) cases.

Case one. $x^{(i)} \in \Omega \cap \bar{\Omega}_{+}^{(i)}(t)$.

By the definition of $\kappa$ it follows that

$$
u_{i}(t, x) \leq e^{\varkappa_{i}} v_{i}(t, x) \quad \text { for all } x \in \bar{\Omega} \quad \text { and } \quad u_{i}\left(t, x^{(i)}\right)=e^{\varkappa_{i}} v_{i}\left(t, x^{(i)}\right),
$$

and

$$
\begin{array}{r}
\frac{d}{d t}\left(\ln \frac{u_{i}\left(t, x^{(i)}\right)}{v_{i}\left(t, x^{(i)}\right)}\right)=\frac{\frac{d}{d t} u_{i}\left(t, x^{(i)}\right)}{u_{i}\left(t, x^{(i)}\right)}-\frac{\frac{d}{d t} v_{i}\left(t, x^{(i)}\right)}{v_{i}\left(t, x^{(i)}\right)} \\
=\frac{\Delta u_{i}\left(t, x^{(i)}\right)}{u_{i}\left(t, x^{(i)}\right)}-\frac{\Delta v_{i}\left(t, x^{(i)}\right)}{v_{i}\left(t, x^{(i)}\right)}+f_{i}\left(t, x^{(i)}, u\left(t, x^{(i)}\right)\right)-f_{i}\left(t, x_{i}, v\left(t, x^{(i)}\right)\right) .
\end{array}
$$


By (3.5),

$$
\frac{\Delta u_{i}\left(t, x^{(i)}\right)}{u_{i}\left(t, x^{(i)}\right)}-\frac{\Delta v_{i}\left(t, x^{(i)}\right)}{v_{i}\left(t, x^{(i)}\right)}=\frac{\Delta\left(u_{i}\left(t, x^{(i)}\right)-e^{\varkappa_{i}} v_{i}\left(t, x^{(i)}\right)\right)}{u_{i}\left(t, x^{(i)}\right)} \leq 0 .
$$

Hence

$$
\frac{d}{d t}\left(\ln \frac{u_{i}\left(t, x^{(i)}\right)}{v_{i}\left(t, x^{(i)}\right)}\right) \leq f_{i}\left(t, x^{(i)}, u\left(t, x^{(i)}\right)\right)-f_{i}\left(t, x^{(i)}, v\left(t, x^{(i)}\right)\right) .
$$

Case two. $x^{(i)} \in \Omega \cap \bar{\Omega}_{-}^{(i)}(t)$.

In a similar manner we prove that

$$
\frac{d}{d t}\left(\ln \frac{v_{i}\left(t, x^{(i)}\right)}{u_{i}\left(t, x^{(i)}\right)}\right) \leq f_{i}\left(t, x^{(i)}, v\left(t, x^{(i)}\right)\right)-f_{i}\left(t, x^{(i)}, u\left(t, x^{(i)}\right)\right) .
$$

Case three. $x^{(i)} \in \partial \Omega \cap \bar{\Omega}_{+}^{(i)}(t)$

This implies that $u_{i}(t, x) \leq e^{\varkappa_{i}} v_{i}\left(t, x^{(i)}\right)$ for all $x \in \Omega$ and $u_{i}\left(t, x^{(i)}\right)=$ $e^{\varkappa_{i}} v_{i}\left(t, x^{(i)}\right)$. Denote $g(x):=u_{i}(t, x)-e^{\varkappa_{i}} v_{i}(t, x)$. The restriction $\left.g\right|_{\partial \Omega}$ of $g$ to $\partial \Omega$ has largest value at the point $x^{(i)}$, consequently $\nabla\left(\left.g\right|_{\partial \Omega}\right)\left(x^{(i)}\right)=0$. By the definition of $g$ it follows that $\frac{\partial g}{\partial \nu}=\frac{\partial u_{i}}{\partial \nu}-e^{\varkappa_{i}} \frac{\partial v_{i}}{\partial \nu}=0$. Hence $\nabla g\left(x^{(i)}\right)=0$.

As $g$ attains its maximum at $x^{(i)}$, this implies that $\Delta g\left(x^{(i)}\right) \leq 0$. Therefore

$$
\begin{array}{r}
\frac{d}{d t}\left(\ln \frac{u_{i}\left(t, x^{(i)}\right)}{v_{i}\left(t, x^{(i)}\right)}\right)=\frac{\frac{d}{d t} u_{i}\left(t, x^{(i)}\right)}{u_{i}\left(t, x^{(i)}\right)}-\frac{\frac{d}{d t} v_{i}\left(t, x^{(i)}\right)}{v_{i}\left(t, x^{(i)}\right)} \\
=\frac{\Delta u_{i}\left(t, x^{(i)}\right)}{u_{i}\left(t, x^{(i)}\right)}-\frac{\Delta v_{i}\left(t, x^{(i)}\right)}{v_{i}\left(t, x^{(i)}\right)} \\
+f_{i}\left(t, x^{(i)}, u\left(t, x^{(i)}\right)\right)-f_{i}\left(t, x^{(i)}, v\left(t, x^{(i)}\right)\right) \\
\leq f_{i}\left(t, x^{(i)}, u\left(t, x^{(i)}\right)\right)-f_{i}\left(t, x^{(i)}, v\left(t, x^{(i)}\right)\right) .
\end{array}
$$

Case four. $x^{(i)} \in \Omega \cup \bar{\Omega}_{-}^{(i)}(t)$

In a similar manner we prove that

$$
\frac{d}{d t}\left(\ln \frac{v_{i}\left(t, x^{(i)}\right)}{u_{i}\left(t, x^{(i)}\right)}\right) \leq f_{i}\left(t, x^{(i)}, v\left(t, x^{(i)}\right)\right)-f_{i}\left(t, x^{(i)}, u\left(t, x^{(i)}\right)\right) .
$$

The cases 1 - 4 with (3.4) give

$$
\begin{array}{r}
D^{+} \Theta_{i}(t) \leq \sum_{i=1}^{N} \max \left\{\sup \left\{f_{i}\left(t, x^{(i)}, u\left(t, x^{(i)}\right)\right)-f_{i}\left(t, x^{(i)}, v\left(t, x^{(i)}\right)\right): x^{(i)} \in \bar{\Omega}_{+}^{(i)}\right\},\right. \\
\left.\sup \left\{f_{i}\left(t, x^{(i)}, u\left(t, x^{(i)}\right)\right)-f_{i}\left(t, x^{(i)}, v\left(t, x^{(i)}\right)\right): x^{(i)} \in \bar{\Omega}_{-}^{(i)}\right\}\right\} .
\end{array}
$$

By assumptions (A3), (A4) and (A5), it follows that

$$
\begin{array}{r}
\left.f_{i}\left(t, x^{(i)}, u_{1}\left(t, x^{(i)}\right), \ldots, u_{N}\left(t, x^{(i)}\right)\right)-f_{i}\left(t, v_{1}\left(t, x^{(i)}\right)\right), \ldots, v_{N}\left(t, x^{(i)}\right)\right) \\
\leq-\underline{b}_{i i}\left(u_{i}\left(t, x^{(i)}\right)-v_{i}\left(t, x^{(i)}\right)\right)+\sum_{\substack{j=1 \\
j \neq i}}^{N} \bar{b}_{i j}(\varepsilon)\left|u_{j}\left(t, x^{(i)}\right)-v_{j}\left(t, x^{(i)}\right)\right|
\end{array}
$$


for each $x^{(i)} \in \bar{\Omega}_{+}^{(i)}(t)$.

By assumptions (A3), (A4) and (A5), it follows that

$$
\begin{array}{r}
\left.f_{i}\left(t, x^{(i)}\right), u_{1}\left(t, x^{(i)}\right), \ldots, u_{N}\left(t, x^{(i)}\right)-f_{i}\left(t, v_{1}\left(t, x^{(i)}\right)\right), \ldots, v_{N}\left(t, x^{(i)}\right)\right) \\
\leq-\underline{b}_{i i}\left(v_{i}\left(t, x^{(i)}\right)-u_{i}\left(t, x^{(i)}\right)\right)+\sum_{\substack{j=1 \\
j \neq i}}^{N} \bar{b}_{i j}(\varepsilon)\left|v_{j}\left(t, x^{(i)}\right)-u_{j}\left(t, x^{(i)}\right)\right|
\end{array}
$$

for each $x^{(i)} \in \bar{\Omega}_{-}^{(i)}(t)$.

Hence

$$
\begin{array}{r}
D^{+} \Theta_{i}(t) \leq \max \left\{\sup _{x^{(i)} \in \bar{\Omega}_{+}^{(i)}}\left\{-\underline{b}_{i i}\left(u_{i}\left(t, x^{(i)}\right)-v_{i}\left(t, x^{(i)}\right)\right)+\sum_{\substack{j=1 \\
j \neq i}}^{N} \bar{b}_{i j}(\varepsilon)\left|u_{j}\left(t, x^{(i)}\right)-v_{j}\left(t, x^{(i)}\right)\right|\right\},\right. \\
\left.\sup _{x^{(i)} \in \bar{\Omega}_{-}^{(i)}}\left\{-\underline{b}_{i i}\left(v_{i}\left(t, x^{(i)}\right)-u_{i}\left(t, x^{(i)}\right)\right)+\sum_{\substack{j=1 \\
j \neq i}}^{N} \bar{b}_{i j}(\varepsilon)\left|v_{j}\left(t, x^{(i)}\right)-u_{j}\left(t, x^{(i)}\right)\right|\right\}\right\}
\end{array}
$$

Since $\delta_{i} \leq u_{i}(t, x), v_{i}(t, x) \leq R_{i}$ for all $i=1, \ldots, N$ and for sufficiently large $t$, using the mean value theorem we have that

$$
\frac{1}{\bar{\delta}}\left|u_{j}(t, x)-v_{j}(t, x)\right| \leq\left|\ln \frac{u_{j}(t, x)}{v_{j}(t, x)}\right| \leq \frac{1}{\underline{\delta}}\left|u_{j}(t, x)-v_{j}(t, x)\right|
$$

for all $t \geq \tilde{T}, x \in \bar{\Omega}, 1 \leq j \leq N$.

Therefore

$$
\begin{array}{r}
D^{+} \Theta_{i}(t) \leq \max \left\{\sup \left\{-\underline{\delta} \underline{b}_{i i}\left|\ln \frac{u_{i}\left(t, x^{(i)}\right)}{v_{i}\left(t, x^{(i)}\right)}\right|+\bar{\delta} \sum_{\substack{j=1 \\
j \neq i}}^{N} \bar{b}_{i j}(\varepsilon)\left|\ln \frac{u_{j}\left(t, x^{(i)}\right)}{v_{j}\left(t, x^{(i)}\right)}\right|: x^{(i)} \in \bar{\Omega}_{+}^{(i)}\right\},\right. \\
\left.\quad \sup \left\{-\underline{\delta} \underline{b}_{i i}\left|\ln \frac{u_{i}\left(t, x^{(i)}\right)}{v_{i}\left(t, x^{(i)}\right)}\right|+\bar{\delta} \sum_{\substack{j=1 \\
j \neq i}}^{N} \bar{b}_{i j}(\varepsilon)\left|\ln \frac{u_{j}\left(t, x^{(i)}\right)}{v_{j}\left(t, x^{(i)}\right)}\right|: x^{(i)} \in \bar{\Omega}_{-}^{(i)}\right\}\right\} \\
\leq\left(-\underline{\delta} \underline{b}_{i i} \sup _{x \in \bar{\Omega}}\left|\ln \frac{u_{i}(t, x)}{v_{i}(t, x)}\right|+\bar{\delta} \sum_{\substack{j=1 \\
j \neq i}}^{N} \bar{b}_{i j}(\varepsilon) \sup _{x \in \bar{\Omega}}\left|\ln \frac{u_{j}(t, x)}{v_{j}(t, x)}\right|\right), \quad t \geq \tilde{T} .
\end{array}
$$

Hence we proved (3.3). 
By (3.1), it follows that

$$
\begin{aligned}
& \sum_{i=1}^{N} \alpha_{i}\left(-\underline{\delta}_{i i} \Theta_{i}(t)+\bar{\delta} \sum_{\substack{j=1 \\
j \neq i}}^{N} \bar{b}_{i j}(\varepsilon) \Theta_{j}(t)\right) \\
= & -\sum_{i=1}^{N} \alpha_{i} \underline{\delta}_{i i} \Theta_{i}(t)+\bar{\delta} \sum_{j=1}^{N} \sum_{\substack{i=1 \\
i \neq j}}^{N} \alpha_{j} \bar{b}_{j i}(\varepsilon) \Theta_{i}(t) \\
= & -\sum_{i=1}^{N}\left(\alpha_{i} \underline{\delta} \underline{b}_{i i}-\sum_{\substack{j=1 \\
j \neq i}}^{N} \alpha_{j} \bar{\delta} \bar{b}_{j i}(\varepsilon)\right) \Theta_{i}(t) \\
\leq & -\epsilon \sum_{i=1}^{N} \Theta_{i}(t), \quad t \geq \tilde{T},
\end{aligned}
$$

where

$$
\epsilon=\min _{1 \leq i \leq N}\left\{\alpha_{i} \underline{\delta} \underline{b}_{i i}-\sum_{\substack{j=1 \\ j \neq i}}^{N} \alpha_{j} \bar{\delta} \bar{b}_{j i}(\varepsilon)\right\}
$$

Hence by (3.3) and (3.7),

$$
D^{+} \Theta(t) \leq \sum_{i=1}^{N} \alpha_{i} D^{+} \Theta_{i}(t) \leq-\epsilon \sum_{i=1}^{N} \Theta_{i}(t) \leq-\frac{\epsilon}{\alpha^{*}} \Theta(t), \quad t \geq \tilde{T}
$$

where $\alpha^{*}=\max \left\{\alpha_{i}: 1 \leq i \leq N\right\}$. Therefore

$$
\Theta(t) \leq \Theta(\tilde{T}) \exp \left(-\frac{\epsilon}{\alpha^{*}}(t-\tilde{T})\right)
$$

for all $t \geq \tilde{T}$.

By (3.6) it follows that

$$
\Theta(t) \geq \alpha_{*} \sum_{i=1}^{N} \Theta_{i}(t) \geq \frac{\alpha_{*}}{\bar{\delta}} \sum_{i=1}^{N} \sup _{x \in \bar{\Omega}}\left|u_{i}(t, x)-v_{i}(t, x)\right|, \quad t \geq \tilde{T},
$$

and

$$
\Theta(\tilde{T}) \leq \alpha^{*} \sum_{i=1}^{N} \Theta_{i}(t) \leq \frac{\alpha^{*}}{\underline{\delta}} \sum_{i=1}^{N} \sup _{x \in \bar{\Omega}}\left|u_{i}(\tilde{T}, x)-v_{i}(\tilde{T}, x)\right|, \quad t \geq \tilde{T}
$$

where $\alpha_{*}=\min \left\{\alpha_{i}: 1 \leq i \leq N\right\}$.

From (3.9), (3.10) and (3.11) we have that

$$
\sum_{i=1}^{N} \sup _{x \in \bar{\Omega}}\left|u_{i}(t, x)-v_{i}(t, x)\right| \leq Z \sum_{i=1}^{N} \sup _{x \in \bar{\Omega}}\left|u_{i}(\tilde{T}, x)-v_{i}(\tilde{T}, x)\right| \cdot \exp (-\gamma(t-\tilde{T})), \quad t \geq \tilde{T},
$$


where $Z=\frac{\bar{\delta} \alpha^{*}}{\underline{\delta} \alpha_{*}}$ and $\gamma=\frac{\epsilon}{\alpha^{*}}(Z$ and $\gamma$ we can take independently on the solutions $u(t, x)$ and $\bar{v}(t, x))$.

By (3.12) it follows that the system $(\mathbb{R})$ is globally attractive and uniformly stable.

\section{Remarks}

Remark 1. Note that conditions (3.1) contain $\underline{\delta}$ and $\bar{\delta}$ which appear in definition of permanence of system $(\mathbb{R})$. In [2] we showed that if we change conditions $\mathrm{ACl}$ to slightly stronger that is

$$
m\left[f_{i}\right]>\sum_{\substack{j=1 \\ j \neq i}}^{N} \frac{\bar{b}_{i j} \bar{a}_{j}}{\underline{b}_{j j}}
$$

then we can estimate $\underline{\delta}$ and $\bar{\delta}$ in terms of parameters of system $(\underline{\mathbb{R}})$. By dissipativity of system $(\underline{\mathrm{R}})$ it follows that we can take $\max _{1 \leq i \leq N}\left\{\frac{\bar{a}_{i}}{\underline{b}_{i i}}\right\}$ as $\bar{\delta}$. Concerning the lower bound (see [2, Theorem 4.1]) as $\underline{\delta}$ we can take

$$
\min _{1 \leq i \leq N}\left\{\frac{1}{\bar{b}_{i i}}\left(\underline{a}_{i}-\sum_{\substack{j=1 \\ j \neq i}}^{N} \frac{\bar{b}_{i j} \bar{a}_{j}}{\underline{b}_{j j}}\right)\right\}
$$

when

$$
\underline{a}_{i}>\sum_{\substack{j=1 \\ j \neq i}}^{N} \frac{\bar{b}_{i j} \bar{a}_{j}}{\underline{b}_{i j}}
$$

for all $1 \leq i \leq N$.

If we define $\underline{\delta}$ and $\bar{\delta}$ as in the above condition (2.1), take a form

$$
\alpha_{i} m\left[f_{i}\right] \geq \sum_{\substack{j=1 \\ j \neq i}}^{N}\left(\alpha_{i} \frac{\bar{b}_{i j} M\left[f_{j}\right]}{\underline{b}_{j j}}+\alpha_{j} \frac{\bar{b}_{j i} M\left[f_{i}\right]}{\underline{b}_{i i}}\right) .
$$

Remark 2.

Definition 5. System ( $(\mathbb{R})$ is uniformly asymptotically stable on $\left[t_{1}, \infty\right)$ if it is uniformly stable and if there is a $r>0$ so that for every $\varepsilon>0$ there is a $T(\varepsilon)>0$ so that for any two positive solutions $u(t, x)=\left(u_{1}(t, x), \ldots, u_{N}(t, x)\right)$ and $v(t, x)=\left(v_{1}(t, x), \ldots, v_{N}(t, x)\right)$ if $t_{2} \geq t_{1}$ and $\left\|u\left(t_{2}, x\right)-v\left(t_{2}, x\right)\right\|<r$ then $\|u(t, x)-v(t, x)\|<\varepsilon$ for $t \geq t_{2}+T(\varepsilon)$.

Note that from attractivity and uniform stability of system $(\underline{\mathbb{R}})$ it follows that system $(\mathbb{R})$ is uniformly asymptotically stable. 


\section{Acknowledgment}

The author thanks Professor Janusz Mierczyński for helpful discussions during writing of this manuscript.

\section{References}

[1] S. Ahmad and A.C. Lazer, Average conditions for global asymptotic stability in a nonautonomous Lotka-Volterra system. onlinear Anal.40(2000) 37-49.

[2] J. Balbus, J. Mierczyński, Time-Averaging and Permanence in Nonautonomous Competitive Systems of PDEs via Vance-Coddington Estimates, Discrete and continuous dynamical systems series B, Vol. 17, No. 5 (2012) doi:10.3934/dcdsb.2012.17.xx

[3] K. Gopalsamy, Global asymptotic stability in an almost - periodic Lotka Volterra system, J. Aust. Math. Soc. Ser B 27 (1986) 346 - 360.

[4] K. Gopalsamy, Global asymptotic stability in a periodic Lotka - Volterra system, J. Aust. Math. Soc. Ser B 27 (1986) $66-72$.

[5] J.A. Langa, J.C. Robinson, A. Suarez, Pullback permanence in a nonautonomous competitive Lotka - Volterra model, J.Differential Equations 190 (2003) $214-238$.

[6] J. Pętela (J. Balbus), Average conditions for Kolmogorov systems, Appl. Math and Comp. 215(2009) $481-494$.

[7] R. Redheffer, Nonautonomous Lotka-Volterra systems. I, J. Differential Equations 127 (1996), no. 2, 519 - 541.

[8] Sze-Bi Hsu, A survey of constructing Lyapunov functions for mathematical models in population biology Taiwanese Journal of mathematics Vol. 9 No 2, June 2005, pp.151-173

[9] A. Tineo, On the asymptotic behavior of some population models. J. Math. Anal. Appl. 167 (1992) 516 - 529.

[10] A. Tineo, J. Riviero, Permanence and asymptotic stability for competitive and Lotka-Volterra systems with diffusion Nonlinear Analysis: Real World Applications 4 (2003), 615-624. 